P256 (continued)

Objective: A smartphone app is potentially useful for delivery of the Special Supplemental Nutrition Program for Women, Infants, and Children (WIC) nutrition education, as many participants use smartphones. It is important to investigate cultural differences that may impact optimal utility, design, and use of a WIC app. The objective of the study was to examine cultural differences regarding the usage of a WIC app prototype designed to improve health behaviors.

Design, Setting, and Participants: A total of 54 WIC mothers in 6 focus groups held in Southwest Texas (79\% Hispanic and $42 \%$ Spanish-only speakers) tested a WIC app prototype. Prompts based on the Unified Theory of Acceptance and Use of Technology (UTAUT) model for behavior intention were included in the focus group discussion guide. Comments were recorded and transcribed. Using Nvivo 10, comments were coded for behavioral intention.

Outcome Measures and Analysis: Emerging themes for behavior intention were established. Observations between English and Spanish speaking groups were compared and differences identified.

Results: Major differences were seen in motivations to use the app and how the app would be used for the family. Spanish speakers viewed smartphones as tools, whereas English speakers habitually used smartphones. English speakers uniquely wanted an app to monitor individual family members' behaviors.

Conclusions and Implications: Differences in WIC app acceptability could be explained by perceptions regarding family structure, the necessity of a smartphone, and habits for seeking emotional and social support. Such differences are important when considering the delivery of electronic nutrition education as well as potential limitations of applying UTAUT to Spanish speaking populations. Funding: Texas Department of State Health Services

\section{P257 Diet Tracking Mobile App Ownership Is Not Associated With Lower Weight, Energy Intake, or Improved Weight Loss Behaviors}

Gabrielle Turner-McGrievy,PhD, MS, RD, brie@sc.edu, University of South Carolina, 915 Greene Street, Columbia, SC 28201; C. Singletary, MPH, USC;

A. Boutte, MPH; C. Dunn, MS, RD; S. Wilcox, PhD

Objective: Dietary self-monitoring using mobile technology holds promise as a way to help people consume fewer calories, engage in effective weight loss behaviors, and improve self-efficacy around weight loss; however, few studies have examined this. The purpose of this study was to determine if installation of dietary mobile apps is linked to weight.

Design, Setting, and Participants: Overweight adults $(\mathrm{n}=101$, BMI $34.1 \pm 5.7 \mathrm{~kg} / \mathrm{m} 2,47.8 \pm 12.0$ years old, $16 \%$ male, 19\% African American) who were recruited to participate in a technology-based mobile weight loss intervention completed surveys and anthropometric assessment at baseline.
Outcome Measures and Analysis: Participants had their height/weight assessed, were asked if they had an app installed on their phone to track diet, and completed the Eating Behavior Inventory (EBI), Weight Efficacy Lifestyle Questionnaire (WEL-Q), and two days of dietary recall.

Results: About half $(n=58)$ of participants reported having installed a diet app. There was no difference in gender or race between diet app users and non-users; however app users were significantly younger $(44.2 \pm 11.9 \mathrm{y})$ than nonusers $(52.6 \pm 10.5 \mathrm{y} ; \mathrm{p}<0.001)$. Age-adjusted models showed no differences in kcals/day $(2176 \pm 833$ non-users vs. $2209 \pm 871$ users; $\mathrm{p}=0.90)$, EBI score $(74.6 \pm 11.4$ nonusers vs. $73.9 \pm 11.0$ users; $p=0.58$ ), or any of the five domains of the WEL-Q (p's all $>0.05$ ).

Conclusions and Implications: The results of this study demonstrate that installing diet tracking apps, without additional behavioral support, may not be enough to impact BMI, energy intake, effective weight loss strategies, or weight loss self-efficacy. These findings point to the need for effective behavioral strategies to be included within apps designed for dietary self-monitoring.

Funding: $\mathrm{NIH}$

\section{P258 Development and Application of Web- Based Dietary Evaluation System Using Food Frequency Questionnaire for Korean Adolescents}

Seungmin Lee,PhD, sullenelee@gmail.com, Sungshin Women's University, \#A-747, 55 Dobongro76gagil, Kangbuk-gu, Seoul, 01133, South Korea; J. Yum, MS; E. Soon $L y u, P h D$, Pukyong National University

Objective: We previously developed a dish-based semiquantitative food frequency questionnaire (FFQ) for Korean adolescents and the developed FFQ's reliability and validity was found reasonable. The objective of the current study was to construct web-based dietary evaluation system applying the FFQ for Korean adolescents and examine its applicability in the context of reliability and validity.

Design, Setting, and Participants: A web-based dietary evaluation system was designed in a comprehensive approach, incorporating not only dietary data survey but also up-to-date nutrition information and individualized eating behavior guidelines. A convenience sample of 50 boys and girls aged 12-18 years agreed in the study participation and completed the FFQ twice and 3 days of dietary recall on the developed website during a two-month period.

Outcome Measures and Analysis: The FFQ's reliability and validity was examined using correlation and cross classification analysis. We also measured participants' subjective levels of the web site's usability, visual effect, understanding, and familiarity.

Results: Spearman correlation coefficients for reliability ranged from 0.74(for vitamin A) to 0.94(for energy). From cross-classification analyses, the proportion of 